\title{
Undifferentiated Pancreatic Carcinoma
}

National Cancer Institute

\section{Source}

National Cancer Institute. Undifferentiated Pancreatic Carcinoma. NCI Thesaurus. Code C5722.

A carcinoma with poor prognosis that arises from the pancreas. It is characterized by the presence of a significant malignant component that does not show differentiation. The malignant cells represent a mixture of large, pleomorphic cells and giant cells, or adenocarcinoma cells and spindle cells, or spindle cells. 